\title{
OPEN System introduction and evaluation of the first Chinese chest EIT device for ICU applications
}

\author{
Shuo-Yao $\mathrm{Qu}^{1,4}$, Meng Dai ${ }^{2,4}$, Shuo Wu ${ }^{1}$, Zhi-Rang $\mathrm{Lv}^{3}$, Xin-Yu Ti ${ }^{1 \bowtie}$ \& Feng Fu ${ }^{2 \bowtie}$
}

Chest electrical impedance tomography (EIT) is a promising application which is used to monitor the ventilation and perfusion of the lung at the bedside dynamically. The aim of the study was to introduce the first Chinese made chest EIT device for ICU application (Pulmo EIT-100). The system design of the hardware and software was briefly introduced. The performance of the system was compared to PulmoVista 500 (Dräger Medical) in healthy volunteers. The EIT system Pulmo EIT-100 consists of impedance measurement module, power supply module, PC all-in-one machine, medical cart and accessories. The performance of the system current source and voltage measurement unit was tested. A total of $\mathbf{5 0}$ healthy lung volunteers were prospectively examined. Subjects were asked to perform repetitive slow vital capacity (SVC) maneuvers with a spirometer. EIT measurements were performed in the following sequence during each SVC with: (1) Pulmo EIT-100, (2) PulmonVista500, (3) Pulmo EIT-100 and (4) PulmonVista500. Linearity and regional ventilation distribution of the reconstructed images from two devices were compared. The output frequency stability of the current source was $2 \mathrm{ppm}$. The amplitude error within one hour was less than $0.32 \%$. The output impedance of the current source was about $50 \mathrm{k} \Omega$. The signal-to-noise ratio of each measurement channel was $\geq 60 \mathrm{~dB}$. For fixed resistance measurements, the measured values drifted about $0.08 \%$ within one hour. For human subjects, the correlations between the spirometry volume and EIT impedance from two devices were both $0.99 \pm 0.01$. No statistical significances were found in the parameters investigated. The repeatability (variability) of measures from the same device was comparable. Our EIT device delivers reliable data and might be used for patient measurement in a clinical setting.

Electrical impedance tomography (EIT) is a non-invasive, radiation-free clinical imaging technique ${ }^{1}$. Chest EIT is one of the most promising application of such technique, which is used to monitor the ventilation and perfusion in real-time at the bedside ${ }^{2}$. A total of 16 or 32 electrodes in form of electrode belt or similar is attached to the thorax surface, usually at the 4 th or 5 th intercostal space. Small insensible alternative currents are injected to the thorax and the corresponding surface voltages are measured. Relative impedance changes are reconstructed accordingly, representing the change of lung volume within the measurement plane.

Nearly 4 decades have passed since the emerge of the technique, only a few commercial devices are available in the market including PulmoVista 500 (Dräger Medical, Lübeck, Germany), Enlight 1800 (Timpel, São Paulo, Brazil), AirTom (Bilab Healthcare, Seoul, Korea) and LuMon (Sentec, Therwil, Switzerland). Therefrom only one is available in China. Nevertheless, the clinical research and daily usage of chest EIT in China are intensive . $^{3}$ Especially due to the outbreak of COVID-19, a Chinese guideline on COVID-19 treatment was issued and EIT was mentioned as one of the mean to titrate PEEP. A recent randomized controlled trial has demonstrated that EIT-guided titration of positive end-expiratory pressure could significantly improve the survival rate in moderate to severe acute respiratory distress syndrome compared to pressure-volume loop ${ }^{4}$. Hence, more and more hospitals and doctors are interested in this technique. Unfortunately, the price of the commercial device in China

${ }^{1}$ Department of Pulmonary and Critical Care Medicine, Xijing Hospital, Fourth Military Medical University, No. 127 Changle West Road, Xi'an 710032, China. ${ }^{2}$ Department of Biomedical Engineering, Fourth Military Medical University, No. 169 Changle West Road, Xincheng District, Xi'an 710032, China. ${ }^{3}$ Northwest Machine, Xi'an, China. ${ }^{4}$ These authors contributed equally: Shuo-Yao Qu and Meng Dai. ${ }^{\square}$ email: tixy_pccm@21cn.com; fengfu@ fmmu.edu.cn 


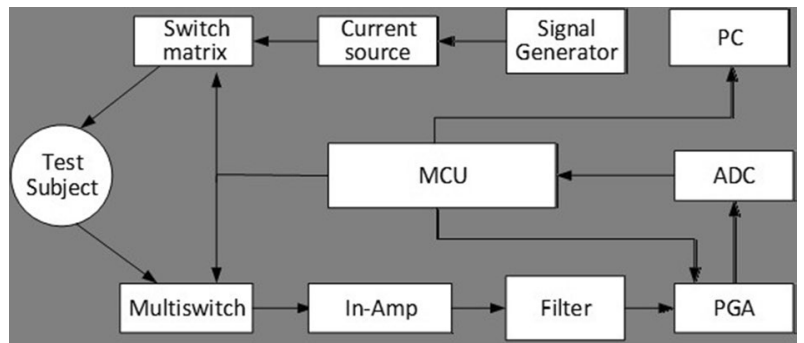

Figure 1. Overall design of the system. $P C$ personal computer, $A D C$ analog-digital converter, $P G A$ programmable gain amplifier, $M C U$ microcontroller unit, $I n$-Amp instrumentation amplifier.

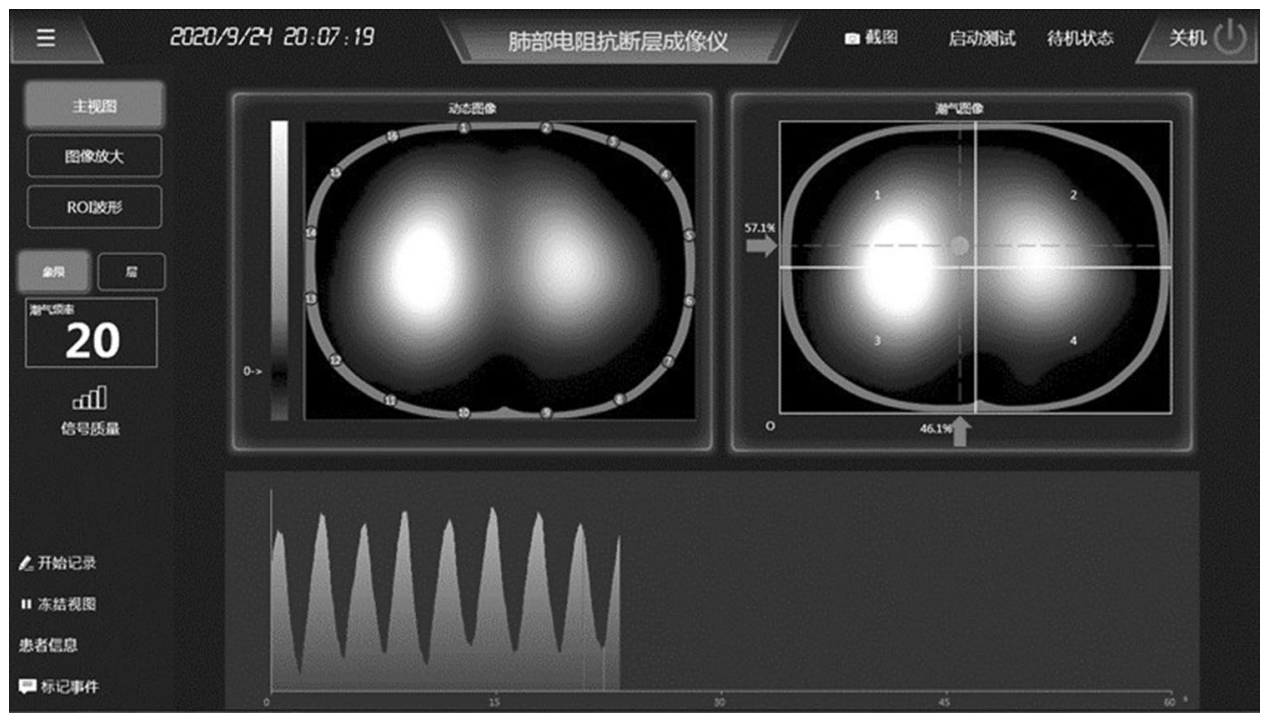

Figure 2. Screenshot of the software display.

is too expensive for most of the hospitals to afford, especially no reimbursements of the device and measurements are possible at the moment, which prohibits the widely use of chest EIT in clinical practice.

Our group has recently developed the first Chinese chest EIT device for ICU applications. In the present study, the system design of the hardware and software was briefly introduced. The performance of the system was compared to PulmoVista 500 in healthy volunteers.

\section{Methods}

System architecture. The EIT system Pulmo EIT-100 (Fourth Military Medical University, FMMU, Xi'an, China) consists of five main parts, which are impedance measurement module, power supply module, PC allin-one machine, medical cart and accessories. The impedance measurement module is the main part of the system, including the control system unit, communication interface management unit, current source, and voltage measurement unit. The EIT system contains 16 measurement channels. The overall functions and configuration of the system are controlled through a PC. The main board contains two processors. One processor is responsible for data pre-processing and communication. The other one is for current signal generation and voltage measurement. The overall design is illustrated in Fig. 1.

PC and software introduction. The PC with Ethernet port (RJ45) is used as the imaging and control device. The PC exchanges commands and data with the lower computer through the high-speed Ethernet port. Our human-machine interface mainly displays the lung impedance time difference image, and the region of interest can be selected for sub-regional display of the impedance waveform. A screenshot of our software display is shown in Fig. 2.

Measurement board main control unit. The function of the measurement main board (PCB) is mainly completed by MCU and FPGA control. The former is responsible for the overall scheduling of the lower computer system. It establishes communication with the PC to receive commands and configuration information from the upper computer. It also controls the FPGA for the operation of the measurement function and transmits the measurement information from the FPGA to the PC for image reconstruction. 


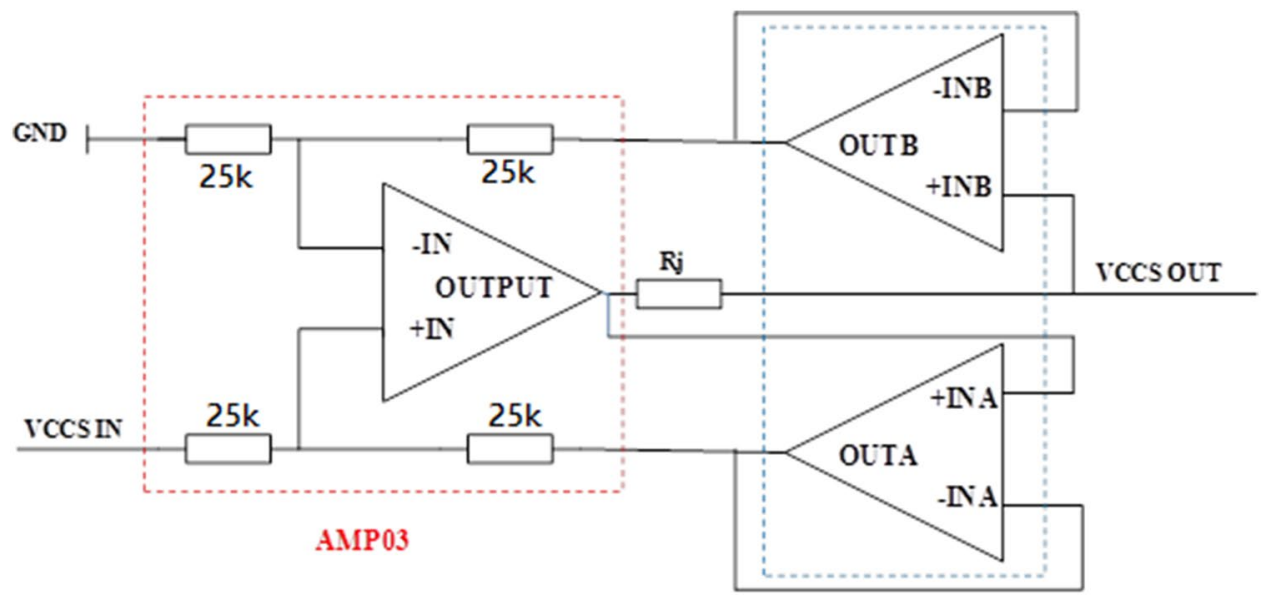

THS4032

Figure 3. Illustration of the VCCS module circuit principle.

Current generator. The demodulation method of PulmoEIT-100 is digital. The current generation unit adopts the driving form of dual current sources, which generate $50-200 \mathrm{kHz}$ single-frequency sine wave current source with a phase difference of $180^{\circ}$. There is a $20 \mathrm{MHz}$ synchronous clock source inside the FPGA to provide synchronous clock signal to other modules. In total it has 16 channels and the frame rate can be up to $50 \mathrm{fps}$.

Th VCCS module circuit principle is illustrated in Fig. 3. The V/I converter can achieve accurate constant current output with wide bandwidth and high output impedance. The signal output from DAC is buffered and filtered into VCCS module for voltage source to current source conversion. The system adopts three op-amps VCCS circuit (the selected op-amps are AMP03 and TH4032). The working bandwidth of AMP03 is 3 MHz. Its amplification setting resistors are built-in, which can better ensure the accuracy of constant current source.

Voltage acquisition unit. The voltage acquisition unit consists of differential amplifier, bandpass filter and gain amplifier. It converts the differential voltage between a pair of electrodes into a single-ended signal. Further, it carries out band-pass filtering to minimize DC component and high-frequency noise to ensure the accuracy and stability of the acquired signal.

System performance evaluation. The performance of the system current source and voltage measurement unit was tested. For the current source, the frequency stability, current stability and output impedance of the output current signal were examined. For the voltage measurement unit, the signal-to-noise ratio (SNR) and stability were examined. To be specific, the device was connected to resistive network and the resistance between every pair channel was $100 \Omega$. The injected current was set to $5 \mathrm{~mA}$ and $100 \mathrm{kHz}$. Data were recorded continuously for $1 \mathrm{~h}$. The SNR was calculated according to Eqs. (1) and (2):

$$
\begin{gathered}
\mathrm{SNR}_{i}=-20 \lg \left(\frac{\sqrt{\frac{1}{\mathrm{~N}-1} \sum_{\mathrm{n}=1}^{\mathrm{N}}\left(\mathrm{V}_{\mathrm{n}}-\overline{\mathrm{V}}\right)^{2}}}{\overline{\mathrm{V}}}\right) \\
\overline{\mathrm{SNR}}=\frac{1}{c h_{\max }} \sum_{i=1}^{c h_{\text {max }}} \mathrm{SNR}_{i}
\end{gathered}
$$

where $\mathrm{i}$ is the channel number; $\mathrm{N}$ is the total number of testing sample $\left(\mathrm{N}=1000\right.$ in the present study); $V_{n}$ is the voltage of the $n$ testing sample; $\bar{V}$ is the average of $V ; c h_{\max }$ is the number of total channels. The current frequency was tested with Fluke 8845A 6.5 Digit Precision Multimeter (FLUKE, Everett, US).

Further, the system performance was evaluated on human subjects. The study protocol was approved by the ethics committees of the Fourth Military Medical University (KY20213003-1) and all subjects signed the informed consent form before the experiment. A total of 50 healthy lung volunteers were prospectively examined (male:female 33:17; age, $47 \pm 15$ years; height, $167 \pm 8 \mathrm{~cm}$; weight, $66 \pm 9 \mathrm{~kg}$ ). Subjects were asked to perform repetitive slow vital capacity (SVC) maneuvers ${ }^{5}$ with a spirometer (HI-101; CHEST M.I., INC., Tokyo, Japan). EIT measurements were performed in the following sequence during each SVC with: (1) Pulmo EIT-100 from FMMU, (2) PulmonVista500 from Dräger Medical, (3) Pulmo EIT-100 and (4) PulmonVista500. The electrode belts from the devices were attached and detached from the subjects' thorax, $\sim 4$ th intercostal space. The level of the electrode plane was marked to ensure the repeatability of electrode placements for every measurement. Relax stable tidal breathing and functional residual capacity level were observed before the SVC maneuver was conducted. Sampling rate of both devices was set to $50 \mathrm{~Hz}$. GREIT algorithm ${ }^{6}$ was used for offline analysis of EIT data from both devices to eliminate the influence of reconstruction methods. 
Tidal variation images (the difference between end-inspiration and end-expiration) were calculated. Impedance values were normalized to the corresponding SVC in milliliters. The clinically widely EIT parameters were used to evaluate the differences of the measured data ${ }^{7}$. In brief, the following measures are calculated:

(a-b) linearity, i.e. the ratio between the tidal variation and impedance changes obtained during SVC, as well as the correlation between volume and impedance changes in individual subjects. Linear interpolation was applied for spirometry data to match the number of data points recorded by EIT.

(c-d) global ventilation distribution, indicated by the tidal variation in the right and left lungs, as well as in the ventral and dorsal regions.

(e-f) spatial ventilation distribution, indicated by the global inhomogeneity (GI) index ${ }^{8,9}$ and the center of ventilation $(\mathrm{CoV})^{9-11}$.

(g) temporal ventilation distribution, indicated by standard deviation of regional ventilation delay (RVD) $)^{12,13}$.

Statistical analysis. Two-sample equivalence test was conducted used to compare the differences of EIT measures between two devices. Bland-Altman plots were used to illustrate the differences. Differences between two measurements from the same devices were divided by the average to show the repeatability of the maneuver. A p value $<0.05$ was considered statistically significant. EIT data and statistical analysis were performed using MATLAB R2015a (the MathWorks Inc., Natick, MA). Since no information regarding the mean and standard deviation of the EIT measures in healthy volunteers was available, sample size was predefined. The post-hoc power was calculated based on the global ventilation distribution indicated by the tidal variation in the right and left lungs.

Ethics approval. All methods of this study were carried out in accordance with the relevant guidelines and regulations of the Fourth Military Medical University. The study was approved by the Ethics Committee of the First Affiliated Hospital of the Forth Military Medical University (No. KY20213003-1).

\section{Results}

We have selected the frequencies of 50,80,100,120 kHz for current source testing. The output frequency stability of the current source was $2 \mathrm{ppm}$. Under the selected frequency, the sine wave signal was collected and analyzed. The amplitude error within one hour was less than $0.32 \%$. Hence, for a short period of time, the current source output fluctuation error is negligible. The output impedance of the current source was about $50 \mathrm{k} \Omega$.

A resistance network phantom was used to test the voltage measurement unit. The signal-to-noise ratio of each measurement channel was $\geq 60 \mathrm{~dB}$. For fixed resistance measurements, the measured values drifted about $0.08 \%$ within one hour.

On human subjects, the measurements are illustrated in Fig. 4. The evaluated EIT parameters calculated based on the EIT data from PulmoEIT-100 and PulmoVista500 were similar as illustrated in Fig. 5. No statistical significances were found in the parameters. The post-hoc power calculation indicated that with sample size of 50 and $\alpha$ of $0.05,1-\beta$ was 0.97 . In Table 1 , the repeatability (variability) of measures from the same device is summarized. No significant differences were found in the variability as well. The correlations between the spirometry volume and EIT impedance were both equaled to $0.99 \pm 0.01$.

\section{Discussions}

In the present study, we evaluated the first Chinese chest EIT device for ICU. The device performance is comparable to the commercial device as examined in the human subjects.

Since the ill-position problem in EIT reconstruction, the EIT devices now available are all reconstructing time-difference images. That is to say, only relative impedance can be reconstructed. Therefore, it is not possible to directly compare the impedance values of two EIT devices. In a previous study, Zhao et al. compared the EIT image analysis with different reconstruction methods ${ }^{7}$. In their study, instead of comparing absolute impedance values, they compared the linearity of the impedance-time curves and ventilation distribution in the reconstructed images. Similarly, in the present study, we adopted these analyses to reflect the linearity, ventilation distribution including spatial and temporal, repeatability, as well as the reliability associating with lung volume changes. The subjects haven been instructed to perform repeatable SVC maneuver to meet the ATS/ERS guideline $\mathrm{e}^{14}$. So the EIT-based parameters are relatively repeatable (Table 1). However, the tidal breathing before the SVC was hard to control. Hence the variability of TV/SVC was higher than other parameters, except for RVD. The evaluation of RVD was conducted under low-flow maneuver ${ }^{12}$. Although the inspiration time during SVC is longer than normal tidal breathing, yet the length is still shorter than that during low-flow maneuver. Given the fact that the recruited subjects are all lung-healthy volunteers, the average values and the variations obtained in the study for RVD are not representing the tidal recruitment, as suggested in the clinical studies (e.g. ${ }^{15,16}$ ).

A recent paper has summarized the EIT circuits and systems available in the labs for various applications ${ }^{17}$. Based on the data collected in this review paper, our device has a relatively high signal-to-noise ratio and frame rate. Wu et al. has introduced a system for neonatal ventilation monitoring, which has a higher frame rate up to $122 \mathrm{fps}^{18}$. The system from Draeger also has a frame rate of $50 \mathrm{fps}$, which shows no limitation in its ventilation and perfusion monitoring ${ }^{19}$. Besides, most of the papers that introduce novel EIT systems mainly focus on system architecture and phantom experiments. Few studies have evaluated the performance of the systems in comparison with the commercial devices. Since the final aim of the device development is the real-time applications in clinical environment. We consider that the performance of the device on human subjects should be the most important end-point of the paper.

As a limitation of this study, the long term device stability was not examined on the human subjects. Nevertheless, the hardware test of current source and voltage measurements showed that the Pulmo EIT-100 from FMMU 
A

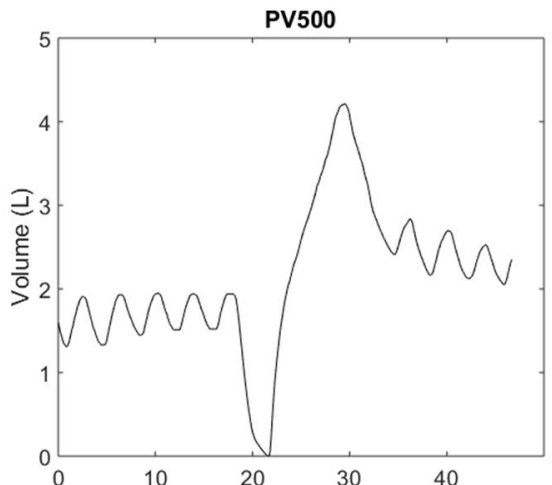

B

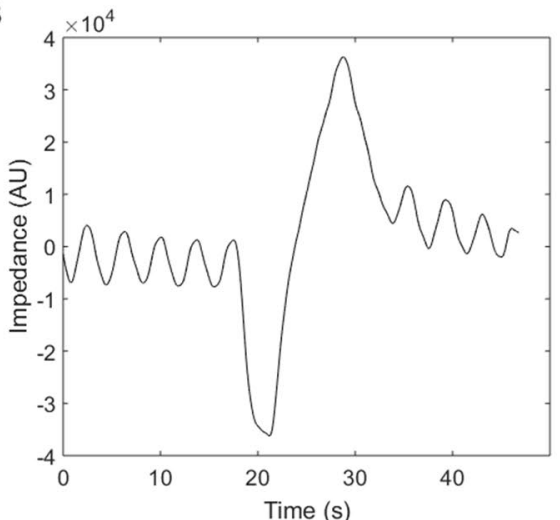

C

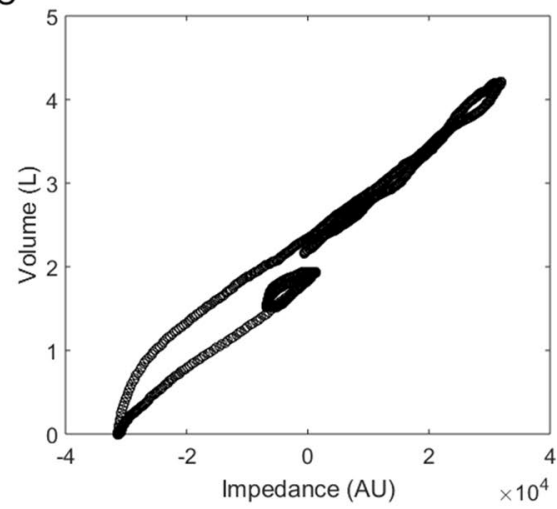

D

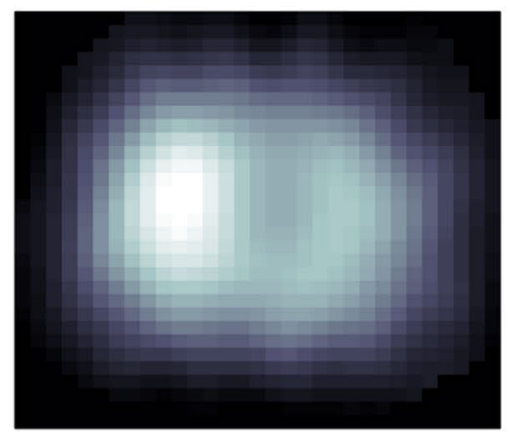

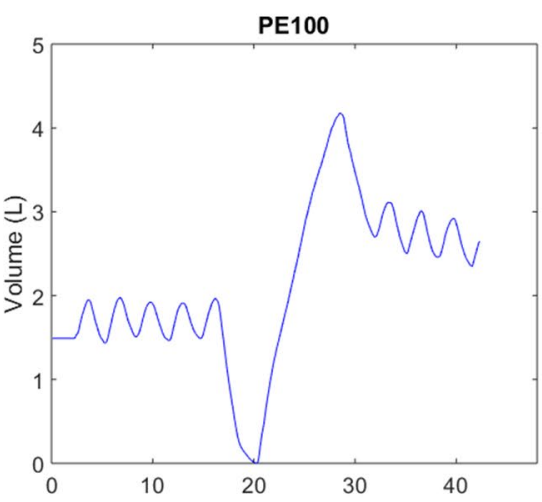
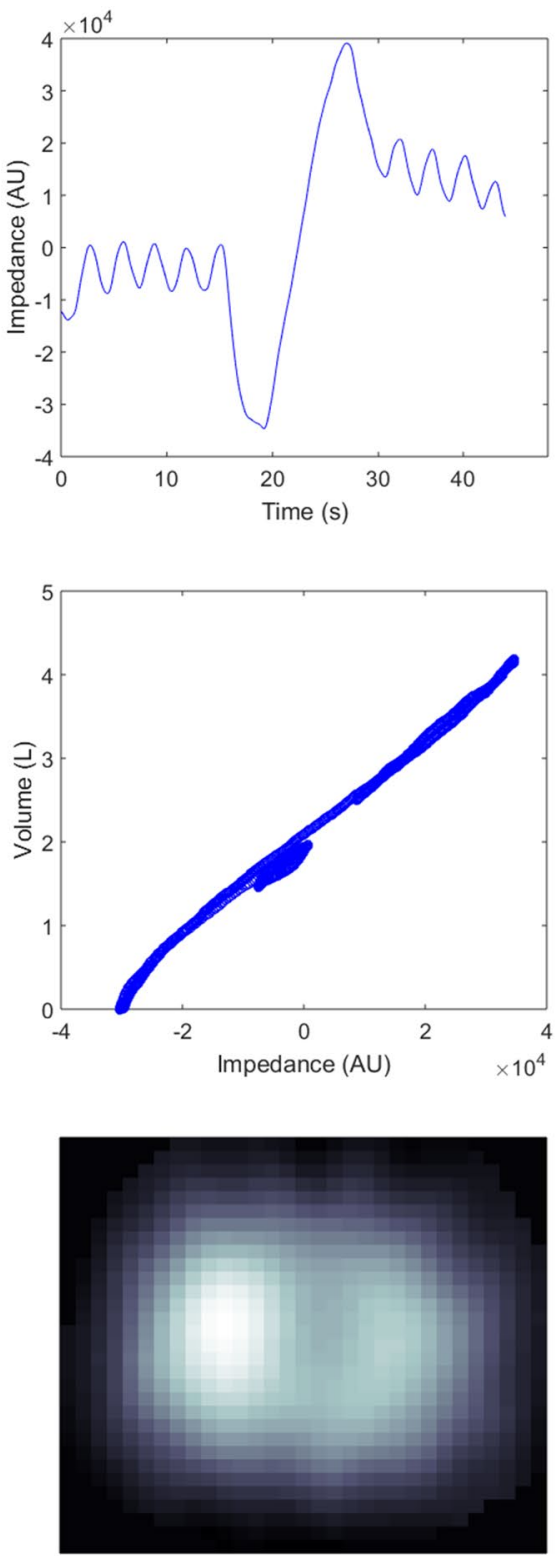

Figure 4. Illustration of the measurement sequence and relative impedance changes. First row, relax tidal breaths and slow vital capacity maneuvers recorded with a spirometer. Second row, impedance-time curves during the SVC maneuver. Third row, correlation between the spirometry volume and EIT impedance changes. Fourth row, impedance variation during SVC. AU, arbitrary unit. PV500 PulmoVista 500, PE100 PulmoEIT-100. 

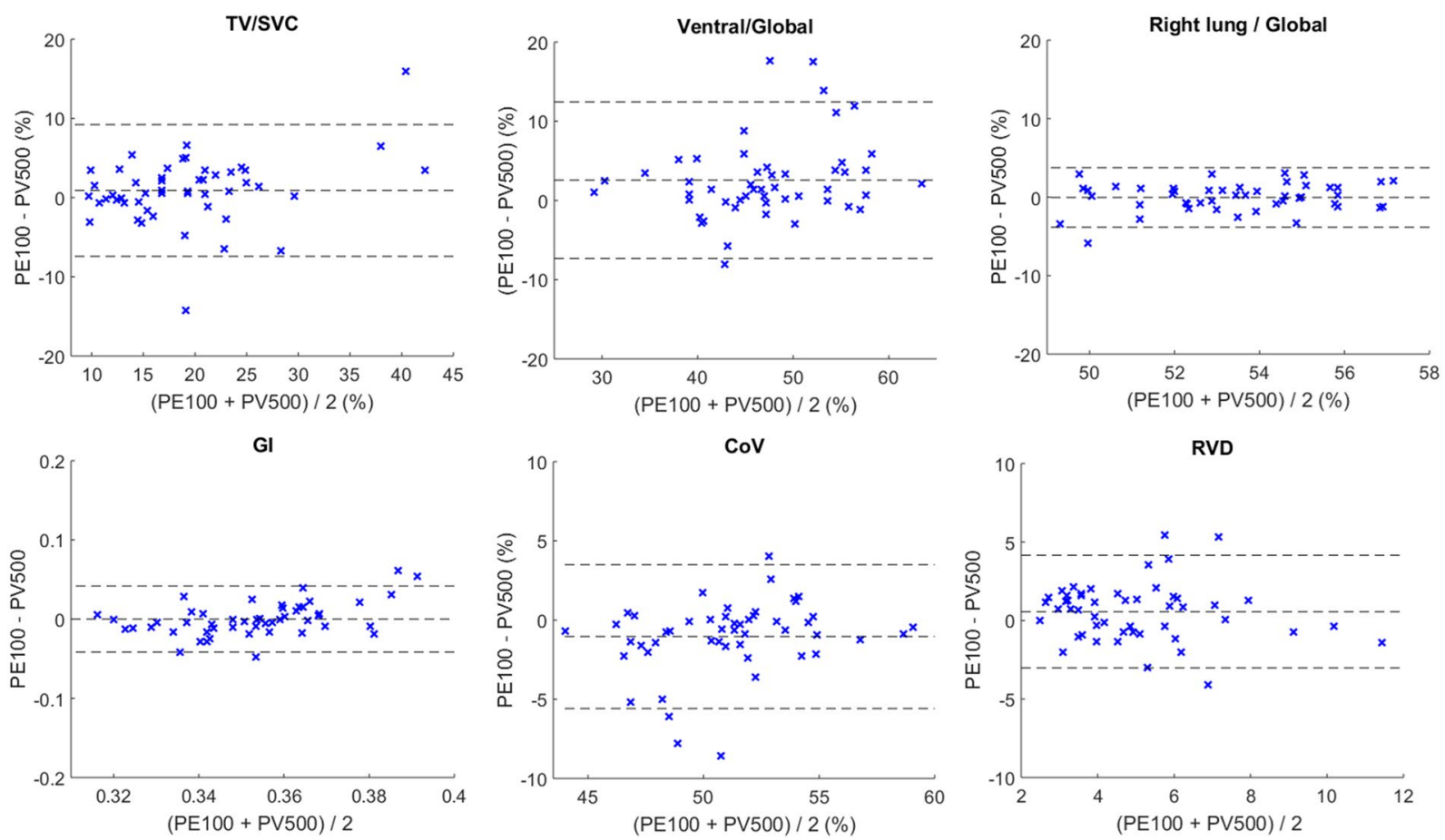

Figure 5. Bland-Altman plots compared the EIT-based measures calculated with PE100 (Pulmo EIT-100, FMMU) and the PV500 (PulmoVista500, Dräger). TV tidal impedance variation, SVC impedance variation during SVC maneuver, GI the global inhomogeneity index, CoV center of ventilation, $R V D$ regional ventilation delay.

\begin{tabular}{|l|l|l|l|}
\hline Variability of repeated measurements with same devices & PulmoEIT100 (\%) & PulmoVista500 (\%) & $\boldsymbol{p}$ \\
\hline TV/SVC & $18.0 \pm 12.0$ & $17.7 \pm 13.7$ & 0.92 \\
\hline Volume-impedance correlation & $0.6 \pm 1.0$ & $0.5 \pm 0.4$ & 0.21 \\
\hline Ventral to global ratio & $5.6 \pm 11.2$ & $4.3 \pm 4.6$ & 0.47 \\
\hline Right lung to global ratio & $2.0 \pm 3.0$ & $1.4 \pm 1.6$ & 0.17 \\
\hline GI & $4.1 \pm 5.4$ & $2.8 \pm 2.5$ & 0.16 \\
\hline CoV & $2.5 \pm 6.4$ & $1.7 \pm 1.9$ & 0.44 \\
\hline RVD & $24.5 \pm 24.3$ & $25.1 \pm 25.1$ & 0.90 \\
\hline
\end{tabular}

Table 1. Variability of EIT measures from the same device. $T V$ tidal impedance variation, $S V C$ impedance variation during SVC maneuver, GI the global inhomogeneity index, $C o V$ center of ventilation, $R V D$ regional ventilation delay.

is stable and reliable for measurements over one hour. The experiment was carried out in only one body position. Nevertheless, the comparison of our system with a widely used commercial product, PulmoVista 500 indicated that the performance of our system was comparable to PulmoVista 500 in respect of image analysis and interpretation. The performance of the proposed system should be further validated in clinical environmental settings.

\section{Conclusion}

The EIT device from FMMU delivers reliable data and might be used for patient measurement in a clinical setting.

\section{Data availability}

The datasets used and/or analyzed in the present study are available from the corresponding author upon reasonable request.

Received: 2 April 2021; Accepted: 13 September 2021

Published online: 29 September 2021

\section{References}

1. Bayford, R. \& Tizzard, A. Bioimpedance imaging: An overview of potential clinical applications. Analyst 137, 4635-4643. https:// doi.org/10.1039/c2an35874c (2012). 
2. Frerichs, I. et al. Chest electrical impedance tomography examination, data analysis, terminology, clinical use and recommendations: Consensus statement of the TRanslational EIT developmeNt stuDy group. Thorax 72, 83-93. https://doi.org/10.1136/thora xjnl-2016-208357 (2017)

3. Zhao, Z., Fu, F. \& Frerichs, I. Thoracic electrical impedance tomography in Chinese hospitals: A review of clinical research and daily applications. Physiol. Meas. 41, 04TR01. https://doi.org/10.1088/1361-6579/ab81df (2020).

4. Hsu, H. J. et al. Positive end-expiratory pressure titration with electrical impedance tomography and pressure-volume curve: A randomized trial in moderate to severe ARDS. Physiol. Meas. 42, 014002. https://doi.org/10.1088/1361-6579/abd679 (2021).

5. Miller, M. R. et al. Standardisation of spirometry. Eur. Respir. J. 26(2), 319-338 (2005).

6. Adler, A. et al. GREIT: A unified approach to 2D linear EIT reconstruction of lung images. Physiol. Meas. 30, S35-55. https://doi. org/10.1088/0967-3334/30/6/S03 (2009).

7. Zhao, Z., Frerichs, I., Pulletz, S., Muller-Lisse, U. \& Moller, K. The influence of image reconstruction algorithms on linear thorax EIT image analysis of ventilation. Physiol. Meas. 35, 1083-1093. https://doi.org/10.1088/0967-3334/35/6/1083 (2014).

8. Zhao, Z., Möller, K., Steinmann, D., Frerichs, I. \& Guttmann, J. Evaluation of an electrical impedance tomography-based global inhomogeneity index for pulmonary ventilation distribution. Intensive Care Med. 35, 1900-1906 (2009).

9. Yang, L. et al. Lung regions identified with CT improve the value of global inhomogeneity index measured with electrical impedance tomography. Quant. Imaging Med. Surg. 11, 1209-1219 (2020).

10. Frerichs, I., Dargaville, P. A., van Genderingen, H., Morel, D. R. \& Rimensberger, P. C. Lung volume recruitment after surfactant administration modifies spatial distribution of ventilation. Am. J. Respir. Crit. Care Med. 174, 772-779 (2006).

11. Frerichs, I. et al. Monitoring perioperative changes in distribution of pulmonary ventilation by functional electrical impedance tomography. Acta Anaesthesiol. Scand. 42, 721-726 (1998).

12. Muders, T. et al. Tidal recruitment assessed by electrical impedance tomography and computed tomography in a porcine model of lung injury*. Crit. Care Med. 40, 903-911. https://doi.org/10.1097/CCM.0b013e318236f452 (2012).

13. Sun, Q. et al. Effects of neurally adjusted ventilatory assist on air distribution and dead space in patients with acute exacerbation of chronic obstructive pulmonary disease. Crit. Care 21, 126. https://doi.org/10.1186/s13054-017-1714-1 (2017).

14. Graham, B. L. et al. Standardization of spirometry 2019 update. An Official American Thoracic Society and European Respiratory Society Technical Statement. Am. J. Respir. Crit. Care Med. 200, e70-e88. https://doi.org/10.1164/rccm.201908-1590ST (2019).

15. Bickenbach, J. et al. Electrical impedance tomography for predicting failure of spontaneous breathing trials in patients with prolonged weaning. Crit. Care 21, 177. https://doi.org/10.1186/s13054-017-1758-2 (2017).

16. Hochhausen, N. et al. Comparison of two experimental ARDS models in pigs using electrical impedance tomography. PLoS One 14, e0225218. https://doi.org/10.1371/journal.pone.0225218PONE-D-19-16039 (2019).

17. Wu, Y., Hanzaee, F. F., Jiang, D., Bayford, R. H. \& Demosthenous, A. Electrical impedance tomography for biomedical applications: Circuits and systems review. IEEE Open J. Circ. Syst. 2, 380-397. https://doi.org/10.1109/ojcas.2021.3075302 (2021).

18. Wu, Y., Jiang, D., Bardill, A., Bayford, R. \& Demosthenous, A. A $122 \mathrm{fps}, 1 \mathrm{MHz}$ bandwidth multi-frequency wearable EIT belt featuring novel active electrode architecture for neonatal thorax vital sign monitoring. IEEE Trans. Biomed. Circ. Syst. 13, 927-937. https://doi.org/10.1109/TBCAS.2019.2925713 (2019).

19. He, H. et al. Influence of overdistension/recruitment induced by high positive end-expiratory pressure on ventilation-perfusion matching assessed by electrical impedance tomography with saline bolus. Crit. Care 24, 586. https://doi.org/10.1186/s13054-02003301-x (2020).

\section{Author contributions}

S.-y.Q. wrote the main manuscript; S.-y.Q. and M.D. collected of the cases and Data statistics; S.W. and Z-r.L. EIT prepared the EIT monitoring; Conception and experiment design: X.-Y.T., F.F. And all authors reviewed the manuscript.

\section{Funding}

This study was partially supported by Grants of National Natural Science Foundation of China (NSFC 51837011 and 52077216), Natural Science Basic Research Program of Shaanxi Province (2020JM-314).

\section{Competing interests}

The authors declare no competing interests.

\section{Additional information}

Correspondence and requests for materials should be addressed to X.-Y.T. or F.F.

Reprints and permissions information is available at www.nature.com/reprints.

Publisher's note Springer Nature remains neutral with regard to jurisdictional claims in published maps and institutional affiliations.

\footnotetext{
(c) (i) Open Access This article is licensed under a Creative Commons Attribution 4.0 International cc) License, which permits use, sharing, adaptation, distribution and reproduction in any medium or format, as long as you give appropriate credit to the original author(s) and the source, provide a link to the Creative Commons licence, and indicate if changes were made. The images or other third party material in this article are included in the article's Creative Commons licence, unless indicated otherwise in a credit line to the material. If material is not included in the article's Creative Commons licence and your intended use is not permitted by statutory regulation or exceeds the permitted use, you will need to obtain permission directly from the copyright holder. To view a copy of this licence, visit http://creativecommons.org/licenses/by/4.0/.
}

(C) The Author(s) 2021 